\title{
GDOP-Assisted Location Estimation Algorithms in Wireless Location Systems
}

\author{
Lin-Chih Chu, Po-Hsuan Tseng, and Kai-Ten Feng \\ Department of Communication Engineering \\ National Chiao Tung University \\ Hsinchu, Taiwan \\ \{iwill.cm95g, walker.cm90\}@nctu.edu.tw and ktfeng@mail.nctu.edu.tw
}

\begin{abstract}
In recent years, wireless location estimation has attracted a significant amount of attention in different areas. The network-based location estimation schemes have been widely adopted based on the radio signals between the mobile station (MS) and the base stations (BSs). The two-step Least Square (LS) method has been studied in related research to provide efficient location estimation of the MS. However, the algorithm results in inaccurate location estimation under the circumstances with poor geometric dilution of precision (GDOP). In this paper, the GDOPassisted location estimation (GOLE) schemes are proposed by considering the geometric relationships between the MS and its associated BSs. According to the minimal GDOP criterion, the BSs are fictitiously repositioned and are served as a new set of BSs within the formulation of the two-step LS algorithm. The proposed GOLE schemes can both preserve the computational efficiency from the two-step LS method and obtain precise location estimation under poor GDOP environments. Comparing with other existing schemes, numerical results demonstrate that the proposed GOLE algorithms can achieve better accuracy in wireless location estimation.
\end{abstract}

Index Terms-Wireless location estimation, geometric dilution of precision (GDOP), time-of-arrival (TOA).

\section{INTRODUCTION}

Wireless location technologies, which are designated to estimate the position of a Mobile Station (MS), have drawn a lot of attention over the past few decades. Different types of location-based services (LBSs) [1] have been proposed and studied, including the emergency 911 (E-911) subscriber safety services, the navigation system, and applications for the wireless sensor networks (WSNs) [2]. Due to the emergent interests in the LBSs, it is required to provide enhanced precision in the location estimation of a MS under different environments.

A variety of wireless location techniques have been studied and investigated. The network-based location estimation schemes have been widely proposed and employed in the wireless communication system. These algorithms locate the position of the MS based on the measured radio signals either from its neighborhood base stations (BSs) in the cellularbased networks or the sensor nodes (SNs) in the WSNs. The major time-based methods for the network-based location

${ }^{1}$ This work was in part funded by the MOE ATU Program 95W803C, NSC 96-2221-E-009-016, MOEA 96-EC-17-A-01-S1-048, the MediaTek research center at National Chiao Tung University, and the Universal Scientific Industrial (USI) Co., Taiwan. estimation techniques are the time-of-arrival (TOA) and the time difference-of-arrival (TDOA). The TOA scheme measures the arrival time of the radio signals coming from different wireless BSs; while the TDOA scheme measures the time difference between the radio signals.

The equations associated with the network-based location estimation schemes are inherently nonlinear. The uncertainties induced by the measurement noises make it more difficult to acquire the MS's estimated position with tolerable precision. Different approaches have been proposed to obtain an approximate location estimation in the previous studies [3] [4]. The Taylor series expansion (TSE) method was utilized in [3] to acquire the location estimation from the time measurements. The scheme requires iterative processes to obtain the location estimate from a linearized system. The major drawback of the TSE method is that it may suffer from the convergence problem due to an incorrect initial guess of the MS's position. The two-step least square (LS) method [4] was adopted as an approximate realization of the maximum likelihood estimator, which does not require iterative processes. It is observed that feasible location estimation can be achieved by adopting these algorithms while the time measurements are symmetrically located w.r.t. the MS. However, asymmetrical measurement inputs to the MS in general result in degraded precision for location estimation. It is especially noticed that this type of situation can frequently occur under non-regular shapes of geometric layouts, e.g. under the randomly distributed sensor networks.

In order to consider the geometric effect to the accuracy of location estimation, the well-known geometric dilution of precision (GDOP) [5] metric can be adopted to facilitate the design of location estimation algorithms. The GDOP is utilized as an index for observing the location precision of the MS under different geometric positions within the networks, e.g. the cellular, the satellite, or the sensor networks. The work in [6] describes the effect and cost resulting from the network topology with significant GDOP values. However, the method for mitigating the GDOP effect has not been extensively addressed in previous studies. The ridge regression signal processing [7] is proposed for reducing the effects of GDOP in the position-fixed navigation systems. Nevertheless, a prefiltered set of initial range measurements is required before the proposed estimation method can be activated. 
In this paper, two GDOP-assisted location estimation (GOLE) algorithms are proposed to enhance the estimation precision by incorporating the GDOP information within the conventional two-step LS algorithm. Based on an initial estimate of the MS's location, the proposed GOLE schemes determine the fictitious locations of the BSs such that the estimated MS will be relocated at a position with the minimal GDOP value. According to the GDOP criterion, the GOLE(1BS) and GOLE(2BS) algorithms are proposed to consider the cases by fictitiously rotating (i.e. not physically relocate) one and two BS's locations respectively. Reasonable location estimation can be acquired within the GOLE algorithms, especially feasible for the cases with poor GDOP circumstances. Simulation results illustrate that the proposed GOLE schemes can achieve higher accuracy for the MS's estimated location compared to the other existing methods.

The remainder of this paper is organized as follows. Section II describes the measurement models and the GDOP metric. The proposed GOLE algorithms are explained in Section III; while Section IV shows the performance evaluation of the proposed schemes. Section V draws the conclusions.

\section{Preliminaries}

\section{A. Measurement Models}

The signal model for the TOA measurements is utilized in this paper. The set $\boldsymbol{r}_{k}$ contains all the available measured relative distance at the $k$ th time step, i.e. $\boldsymbol{r}_{k}=$ $\left\{r_{1, k}, \ldots, r_{i, k}, \ldots, r_{N_{k}, k}\right\}$, where $N_{k}$ denotes the number of available BSs at the time step $k$. The measured relative distance $\left(r_{i, k}\right)$ between the MS and the $i$ th BS (obtained at the $k$ th time step) can be represented as

$$
r_{i, k}=c \cdot t_{i, k}=\zeta_{i, k}+n_{i, k}+e_{i, k} \quad i=1,2, \ldots, N_{k}
$$

where $t_{i, k}$ denotes the TOA measurement obtained from the $i$ th $\mathrm{BS}$ at the $k$ th time step, and $c$ is the speed of light. $r_{i, k}$ is contaminated with the TOA measurement noise $n_{i, k}$ and the non-line-of-sight (NLOS) error $e_{i, k}$. It is noted that the measurement noise $n_{i, k}$ is in general considered as zero mean with Gaussian distribution. On the other hand, the NLOS error $e_{i, k}$ is modeled as exponentially-distributed for representing the positive bias due to the non-line-of-sight effect [8]. The noiseless relative distance $\zeta_{i, k}$ (in (1)) between the MS's true position and the $i$ th $\mathrm{BS}$ can be obtained as

$$
\zeta_{i, k}=\left[\left(x_{k}-x_{i, k}\right)^{2}+\left(y_{k}-y_{i, k}\right)^{2}\right]^{\frac{1}{2}}
$$

where $\boldsymbol{x}_{k}=\left[\begin{array}{ll}x_{k} & y_{k}\end{array}\right]$ represents the MS's true position and $\boldsymbol{x}_{i, k}$ $=\left[\begin{array}{ll}x_{i, k} & y_{i, k}\end{array}\right]$ is the location of the $i$ th BS for $i=1$ to $N_{k}$. Therefore, the set of all the available BSs at the $k$ th time step can be obtained as $\boldsymbol{P}_{B S, k}=\left\{\boldsymbol{x}_{1, k}, \ldots, \boldsymbol{x}_{i, k}, \ldots, \boldsymbol{x}_{N_{k}, k}\right\}$.

\section{B. GDOP Metric}

The GDOP metric is utilized to describe the geometric effect on the relationship between the measurement error and the position determination error [5]. Fig. 1 illustrates the schematic diagram of the network layout for the GDOP computation. In general, a larger GDOP value corresponds

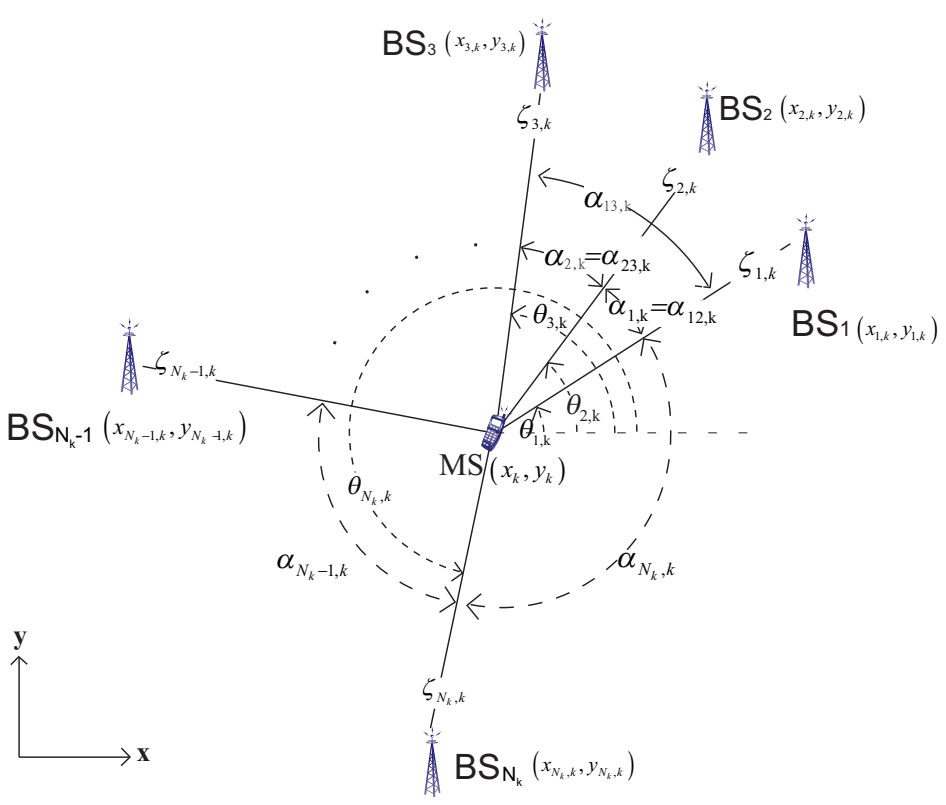

Fig. 1. Schematic diagram of the network layout for GDOP computation.

to a comparably worse geometric layout (established by the MS and its associated BSs), which consequently results in augmented errors for location estimation. On the other hand, as the GDOP value becomes smaller, the effect from the geometric relationship to the location estimation accuracy will turn out to be insignificant. Considering the MS's location under the two-dimensional coordinate, the GDOP value $(\mathrm{G})$ obtained at the MS's true position $\boldsymbol{x}_{k}$ can be represented as

$$
\mathrm{G}_{\boldsymbol{x}_{k}}=\left\{\operatorname{trace}\left[\left(\mathbf{H}_{\boldsymbol{x}_{k}}^{T} \mathbf{H}_{\boldsymbol{x}_{k}}\right)^{-1}\right]\right\}^{\frac{1}{2}}
$$

where

$$
\mathbf{H}_{x_{k}}=\left[\begin{array}{cc}
\frac{x_{k}-x_{1, k}}{\zeta_{1, k}} & \frac{y_{k}-y_{1, k}}{\zeta_{1, k}} \\
\cdots & \ldots \\
\frac{x_{k}-x_{i, k}}{\zeta_{i, k}} & \frac{y_{k}-y_{i, k}}{\zeta_{i, k}} \\
\ldots & \ldots \\
\frac{x_{k}-x_{N_{k}, k}}{\zeta_{N_{k}, k}} & \frac{y_{k}-y_{N_{k}, k}}{\zeta_{N_{k}, k}}
\end{array}\right]
$$

The elements within the matrix $\mathbf{H}_{x_{k}}$ can be acquired from (2). It is noted that (3) associated with (4) are utilized for representing the GDOP metric in most of the research studies. In order to facilitate the design of the proposed GOLE schemes, coordinate transformation is conducted as shown in Fig. 1, i.e. $\boldsymbol{x}_{k}-\boldsymbol{x}_{i, k}=\left[\zeta_{i, k} \cos \theta_{i, k}, \zeta_{i, k} \sin \theta_{i, k}\right]$ for $i=1$ to $N_{k}$, where $\theta_{i, k}$ represents the angle formed by the vector of $\boldsymbol{x}_{k}-\boldsymbol{x}_{i, k}$ w.r.t. the positive $x$-axis. Furthermore, the relative angles $\alpha_{i, k}$ between the neighboring BSs are defined as

$$
\alpha_{i, k}= \begin{cases}\theta_{i+1, k}-\theta_{i, k} & 1 \leq i \leq N_{k}-1 \\ 2 \pi+\theta_{1, k}-\theta_{N_{k}, k} & i=0, N_{k}\end{cases}
$$

It is noted that $i=0$ is utilized for circular counting in order to facilitate the notations that will be utilized in the paper. 


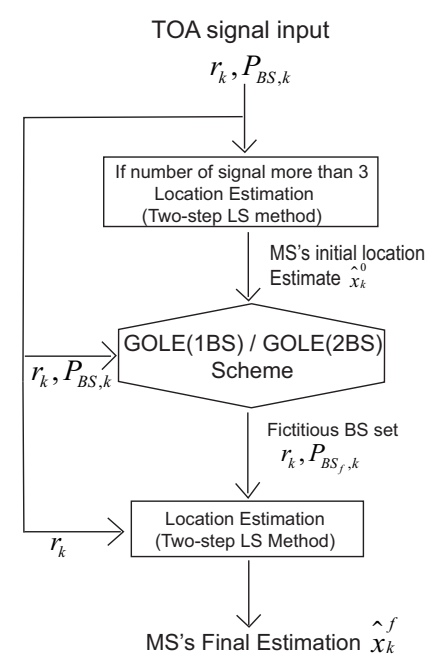

Fig. 2. Schematic diagram of the proposed GOLE algorithms.

Furthermore, the relative angles $\alpha_{p q, k}$ between each arbitrary $p$ th and $q$ th BSs are further defined as

$$
\alpha_{p q, k}=\sum_{\forall p<q}^{q-1} \alpha_{p, k}
$$

where $1 \leq p<N_{k}, 1 \leq q \leq N_{k}$ and $\alpha_{p, k}$ is defined as in (5). Consequently, the GDOP value with the matrix form in (3) can be reformulated as a function of $\alpha_{p q, k}$, which is also a function of $\alpha_{i, k}$, as

$$
\mathrm{G}_{\boldsymbol{x}_{k}}=\left[\frac{N_{k}}{\sum_{q=2}^{N_{k}} \sum_{p=1}^{q-1} \sin ^{2}\left(\alpha_{p q, k}\right)}\right]^{\frac{1}{2}}
$$

In the next section, the results obtained from (7) will be utilized for the design of the proposed GOLE algorithms.

\section{Proposed GDOP-Assisted Location Estimation (GOLE) ALGORITHMS}

The main objective of the proposed GOLE schemes is to enhance the conventional two-step LS algorithm [4] by considering the geometric effect to the location estimation accuracy. Fig. 2 illustrates the schematic diagram of the proposed GOLE algorithms. In order to facilitate the location estimation for the MS, three TOA measurements and the location information of the corresponding BSs are considered available to the MS at the time instant $k$, i.e. $\boldsymbol{r}_{k}=\left\{r_{1, k}, r_{2, k}, r_{3, k}\right\}$ and $\boldsymbol{P}_{B S, k}=\left\{\boldsymbol{x}_{1, k}, \boldsymbol{x}_{2, k}, \boldsymbol{x}_{3, k}\right\}$. With the available information, the two-step LS method can acquire the MS's initial location estimate $\hat{\boldsymbol{x}}_{k}^{o}=\left[\hat{x}_{k}^{o} \hat{y}_{k}^{o}\right]$ within two computing iterations.

The GOLE algorithms are proposed to further enhance the precision of the initial location estimation of the MS. Based on the available measurement information from the BSs, the concept of the proposed GOLE schemes is to acquire the locations of the fictitious BSs such as to attain the minimal GDOP value w.r.t the MS's initial location estimate. At the second phase of the GOLE schemes, the position information of these fictitious BSs will be utilized to replace that of the original
BSs in order to achieve better geometric layout for location estimation. Two GOLE algorithms, i.e. the GOLE(1BS) and GOLE(2BS) schemes, are stated as follows.

\section{A. GOLE(1BS) Scheme}

In order to facilitate the design of the proposed GOLE(1BS) scheme, the property obtained from the GDOP metric is observed and derived. The minimal GDOP value is determined by adjusting one BS's location in Lemma 1 (i.e. with one degree-of-freedom) as follows.

Lemma 1. The MS located at $\boldsymbol{x}_{k}$ is surrounded by three BSs at $\boldsymbol{x}_{i, k}$ (for $i=1$ to 3 ). The angles between every two adjacent $B S s$ to the MS are defined as $\alpha_{i, k}$. It is assumed that only the $\ell$ th BS's location is adjustable; while the positions for the other two BSs are considered fixed. The minimal attainable GDOP occurs as the angle $\alpha_{\ell, k}$ is adjusted to be

$$
\alpha_{\ell, k}^{m}=\frac{1}{2} \tan ^{-1}\left(\frac{-\sin \left(2 \sum_{i=1, i \neq \ell-1, \ell}^{3} \alpha_{i, k}\right)}{\cos \left(2 \sum_{i=1, i \neq \ell-1, \ell}^{3} \alpha_{i, k}\right)+1}\right)
$$

Therefore, the minimal attainable GDOP value w.r.t. $\boldsymbol{x}_{k}$ becomes

$$
\mathrm{G}_{\boldsymbol{x}_{k}}^{m}=\left[\frac{3}{2 \sin ^{2}\left(\alpha_{\ell, k}^{m}\right)+\sum_{i=1, i \neq \ell-1, \ell}^{3} \sin ^{2}\left(\alpha_{i, k}\right)}\right]^{\frac{1}{2}}
$$

Proof: According to (7), it is observed that the GDOP value $\mathrm{G}_{\boldsymbol{x}_{k}}$ w.r.t. $\boldsymbol{x}_{k}$ is regarded as a function of the angles $\alpha_{i, k}$ for all $i=1$ to 3 . Since only the $\ell$ th BS (for $1 \leq \ell \leq 3$ ) is considered adjustable, there is merely one degree-of-freedom that is considered tunable (i.e. $\alpha_{\ell, k}$ ) among all the angles $\alpha_{i, k}$ for $i=1$ to 3 . It is noted that the other angle $\alpha_{\ell-1, k}$, which is also modified due to the movement of the $\ell$ th BS, can be represented as a function of $\alpha_{\ell, k}$, i.e. $\alpha_{\ell-1, k}=2 \pi-\alpha_{\ell, k}-$ $\sum_{i=1, i \neq \ell-1, \ell}^{3} \alpha_{i, k}$. Consequently, the GDOP value as denoted in (7) will only be dependent to the angle $\alpha_{\ell, k}$ as $\mathrm{G}_{\boldsymbol{x}_{k}}\left(\alpha_{\ell, k}\right)$. The angle $\alpha_{\ell, k}^{m}$ which results in the minimal GDOP value can therefore be acquired as

$$
\alpha_{\ell, k}^{m}=\arg \left\{\min _{\forall \alpha_{\ell, k}} \mathrm{G}_{x_{k}}\left(\alpha_{\ell, k}\right)\right\}
$$

It can be observed that (10) can be achieved if the following conditions on the first and second derivatives of $G_{x_{k}}$ are satisfied, i.e.

$$
\begin{gathered}
{\left[\frac{\partial \mathrm{G}_{\boldsymbol{x}_{k}}\left(\alpha_{\ell, k}\right)}{\partial \alpha_{\ell, k}}\right]_{\alpha_{\ell, k}=\alpha_{\ell, k}^{m}}=0} \\
{\left[\frac{\partial^{2} \mathrm{G}_{\boldsymbol{x}_{k}}\left(\alpha_{\ell, k}\right)}{\partial^{2} \alpha_{\ell, k}}\right]_{\alpha_{\ell, k}=\alpha_{\ell, k}^{m}}>0}
\end{gathered}
$$

By solving (11) and (12), the angle $\alpha_{\ell, k}^{m}$ can be computed as in (8). The minimal GDOP value w.r.t. $\boldsymbol{x}_{k}$ can consequently be obtained as in (9).

The GOLE(1BS) scheme is designed to fictitiously relocate the position of one BS according to the minimal GDOP criterion. Without lose of generality, it is considered that 
$\mathrm{BS}_{1}$ (i.e. $\boldsymbol{x}_{1, k}$ ) is the adjustable BS within the GOLE(1BS) scheme. The position of the fictitious $\mathrm{BS}_{1}$ is designed such that the initial estimated MS $\left(\hat{\boldsymbol{x}}_{k}^{o}\right)$ will be located at a minimal GDOP position based on the existing geometric layout $\boldsymbol{P}_{B S, k}=\left\{\boldsymbol{x}_{1, k}, \boldsymbol{x}_{2, k}, \boldsymbol{x}_{3, k}\right\}$. In other words, based on the initial location estimate $\hat{\boldsymbol{x}}_{k}^{o}$ associated with the information coming from the BSs (i.e. $\boldsymbol{r}_{k}$ and $\boldsymbol{P}_{B S, k}$ ), the three relative angles $\alpha_{1, k}$, $\alpha_{2, k}$, and $\alpha_{3, k}$ between the BSs w.r.t. the MS can be obtained. By adopting the results from Lemma 1, the minimal attainable GDOP $G_{\hat{\boldsymbol{x}}_{k}^{o}}$ w.r.t. the MS's initial estimate $\hat{\boldsymbol{x}}_{k}^{o}$ occurs as the angle $\alpha_{1, k}$ is adjusted as

$$
\alpha_{1, k}^{m}=\frac{1}{2} \tan ^{-1}\left(\frac{-\sin \left(2 \alpha_{2, k}\right)}{\cos \left(2 \alpha_{2, k}\right)+1}\right)
$$

It is noted that the angle $\alpha_{2, k}$ between $\mathrm{BS}_{2}$ and $\mathrm{BS}_{3}$ is considered a fixed value; while $\alpha_{3, k}$ is dependent to the variable angle $\alpha_{1, k}$, i.e. $\alpha_{3, k}=\left(2 \pi-\alpha_{2, k}\right)-\alpha_{1, k}$.

As a result, the new set of BSs for location estimation is obtained as $\boldsymbol{P}_{B S_{f}, k}^{(1)}=\left\{\boldsymbol{x}_{f 1, k}, \boldsymbol{x}_{2, k}, \boldsymbol{x}_{3, k}\right\}$, where $\boldsymbol{x}_{f 1, k}$ denotes the location of the fictitious BS as

$$
\begin{aligned}
x_{f 1, k} & =r_{1, k} \cos \left(\theta_{2, k}-\alpha_{1, k}^{m}\right) \\
y_{f 1, k} & =r_{1, k} \sin \left(\theta_{2, k}-\alpha_{1, k}^{m}\right)
\end{aligned}
$$

where $\alpha_{1, k}^{m}$ is obtained from (13). The set of updated locations for the BSs $\boldsymbol{P}_{B S_{f}, k}^{(1)}$ associated with the original TOA measurements $\boldsymbol{r}_{k}=\left\{r_{1, k}, r_{2, k}, r_{3, k}\right\}$ are exploited to conduct the second-phase two-step LS method as shown in Fig. 2. Consequently, the MS's final location estimation $\hat{\boldsymbol{x}}_{k}^{f}=\left[\hat{x}_{k}^{f} \hat{y}_{k}^{f}\right]$ by adopting the proposed GOLE(1BS) scheme can be obtained.

\section{B. GOLE(2BS) Scheme}

For the design of the GOLE(2BS) scheme, the statement and proof for Lemma 2 that regulates all the locations of the BSs (i.e. with three degree-of-freedom) is described as follows.

Lemma 2. The MS located at $\boldsymbol{x}_{k}$ is surrounded by three BSS at $\boldsymbol{x}_{i, k}$ (for $i=1$ to 3 ). The angles between every two adjacent $B S s$ to the MS are defined as $\alpha_{i, k}$. Considering the case that the locations of all the three BSs are adjustable. The minimal GDOP value w.r.t. $\boldsymbol{x}_{k}$ is obtained as $\mathrm{G}_{\boldsymbol{x}_{k}}^{m}=2 / \sqrt{3}$, which occurs as the angles $\alpha_{i, k}$ are regulated to be equivalent with each other as $\alpha_{i, k}^{m}=2 \pi / 3$ for all $i=1$ to 3 .

Proof: It can be observed from (7) that the GDOP value is regarded as a function of the angles $\alpha_{i, k}$ for all $i=1$ to 3 , i.e. $\mathrm{G}_{\boldsymbol{x}_{k}}\left(\alpha_{1, k}, \alpha_{2, k}, \alpha_{3, k}\right)$. By defining $\boldsymbol{\alpha}_{k}=\left[\alpha_{1, k} \alpha_{2, k} \alpha_{3, k}\right]$, the angles $\alpha_{i, k}^{m}$ which result in the minimal GDOP value can therefore be acquired as

$$
\alpha_{i, k}^{m}=\arg \left\{\min _{\forall \alpha_{i, k}} \mathrm{G}_{\boldsymbol{x}_{k}}\left(\boldsymbol{\alpha}_{k}\right)\right\}
$$

for $i=1$ to 3 . Similar to the proof as in Lemma $1, \alpha_{i, k}^{m}$ in (15) can be acquired if

$$
\left[\frac{\partial \mathrm{G}_{\boldsymbol{x}_{k}}\left(\boldsymbol{\alpha}_{k}\right)}{\partial \alpha_{i, k}}\right]_{\boldsymbol{\alpha}_{k}=\boldsymbol{\alpha}_{k}^{m}}=0
$$

$$
\left[\frac{\partial^{2} \mathrm{G}_{\boldsymbol{x}_{k}}\left(\boldsymbol{\alpha}_{k}\right)}{\partial^{2} \alpha_{i, k}}\right]_{\boldsymbol{\alpha}_{k}=\boldsymbol{\alpha}_{k}^{m}}>0
$$

for $i=1$ to 3 . It is noted that $\boldsymbol{\alpha}_{k}^{m} \triangleq\left[\alpha_{1, k}^{m} \alpha_{2, k}^{m} \alpha_{3, k}^{m}\right]$. By solving the set of equations obtained from (16) and (17), the angles $\alpha_{i, k}^{m}$ can be computed as

$$
\alpha_{i, k}^{m}=\frac{2 \pi}{3}
$$

which are considered equivalent for all $i=1$ to 3 . By substituting (18) into (7), the minimal GDOP value can therefore be obtained as

$$
\mathrm{G}_{\boldsymbol{x}_{k}}^{m}\left(\boldsymbol{\alpha}_{k}^{m}\right)=\frac{2}{\sqrt{3}}
$$

In order to achieve the minimal GDOP for the existing geometric layout, the GOLE(2BS) scheme is designed by considering the case while all the BSs are fictitiously movable. By adopting the results from Lemma 2, it can be observed that the three BSs are fictitiously adjusted such that equally partitioned angles are acquired, i.e. $\alpha_{i, k}^{m}=2 \pi / 3$ for $i=1$ to 3 . Therefore, the set of fictitious BSs by exploiting the GOLE(2BS) scheme is represented as $\boldsymbol{P}_{B S_{f}, k}^{(2)}=\left\{\boldsymbol{x}_{f 1, k}, \boldsymbol{x}_{f 2, k}, \boldsymbol{x}_{f 3, k}\right\}$, where $\boldsymbol{x}_{f i, k}$ (for $i=1$ to 3 ) indicates the locations of the fictitious BSs. It is noted that $\boldsymbol{x}_{f 1, k}$ is selected to be $\boldsymbol{x}_{1, k}$ as the rotation reference. Consequently, the locations of the other two fictitious BSs can be acquired as

$$
\begin{aligned}
& x_{f i, k}=r_{i, k} \cos \left(\theta_{1, k}+\sum_{s=1}^{i-1} \alpha_{s, k}^{m}\right) \\
& y_{f i, k}=r_{i, k} \sin \left(\theta_{1, k}+\sum_{s=1}^{i-1} \alpha_{s, k}^{m}\right)
\end{aligned}
$$

for $i=2$ and 3. Similar to the GOLE(1BS) scheme, the fictitious locations of the BSs $\boldsymbol{P}_{B S_{f}, k}^{(2)}$ associated with the TOA measurements $\boldsymbol{r}_{k}$ are utilized to serve as the new set of measurement inputs for the two-step LS method at the second stage. As a result, the final location estimate of the MS (i.e. $\hat{\boldsymbol{x}}_{k}^{f}$ ) can be acquired.

\section{Performance Evaluation}

Simulations are performed to show the effectiveness of the GOLE algorithms under different network topologies and the MS's positions. The proposed GOLE(1BS) and GOLE(2BS) schemes are compared with the exiting two-step LS and the TSE algorithms. As shown in Fig. 3, two different types of geometric layouts are designed to validate the effectiveness of the proposed GOLE algorithms. The left plot illustrates the case while the MS is located at a symmetrical geometric layout, i.e. with smaller GDOP value as $\mathrm{G}_{x_{k}}^{b}=1.44$. On the other hand, a comparable worse geometric layout is designed as shown in the right plot, which results in the GDOP value as $\mathrm{G}_{\boldsymbol{x}_{k}}^{w}=11.08$. It is noted that the minimal GDOP value for a three-BS geometry is obtained as $\mathrm{G}_{\boldsymbol{x}_{k}}^{m}=2 / \sqrt{3}=1.15$. 


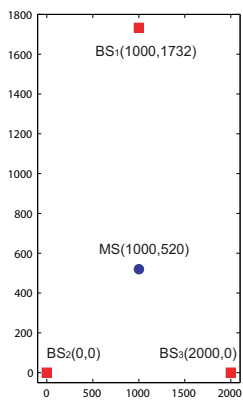

Better Geometric Layout

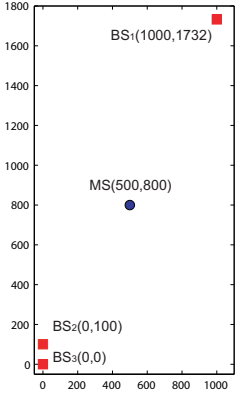

Worse Geometric Layout
Fig. 3. Network topologies for performance evaluation (left plot: better geometric layout with $\mathrm{G}_{x_{k}}^{g}=1.44$; right plot: worse geometric layout with $\left.\mathrm{G}_{x_{k}}^{b}=11.08\right)$.

\section{A. Noise Models}

Different noise models [8] are considered in the simulations in order to represent the environments with both the LOS and the NLOS signals. The model for the measurement noise of the TOA signals is selected as the Gaussian distribution with zero mean and 10 meters of standard deviation, i.e. $n_{i, k} \sim$ $\mathcal{N}(0,100)$. On the other hand, an exponential distribution $p_{e_{i, k}}(\tau)$ is assumed for the NLOS noise model of the TOA measurements as

$$
p_{e_{i, k}}(v)= \begin{cases}\frac{1}{\lambda_{i, k}} \exp \left(-\frac{v}{\lambda_{i, k}}\right) & v>0 \\ 0 & \text { otherwise }\end{cases}
$$

where $\lambda_{i, k}=c \cdot \tau_{i, k}=c \cdot \tau_{m}\left(\zeta_{i, k}\right)^{\varepsilon} \rho$. The parameter $\tau_{i, k}$ is the RMS delay spread between the $i$ th BS to the MS, and $\tau_{m}$ represents the median value of $\tau_{i, k} . \varepsilon$ is the path loss exponent which is assumed to be 0.5 , and the factor for shadow fading $\rho$ is set to 1 in the simulations. It is noted that the parameters for the noise models as listed in this subsection primarily fulfill the environment while the MS is located within the rural area.

\section{B. Simulation Results}

Figs. 4 and 5 illustrates the case for performance comparison under the NLOS environment. It is noted that Fig. 5 is illustrated by observing the RMS errors versus the median values of the NLOS noises (i.e. $\tau_{m}$ ). It can be observed that the proposed GOLE(2BS) scheme outperforms the other algorithms even under the existence of the NLOS errors, i.e. around $250 \mathrm{~m}$ less in RMS error compared to the twostep LS method with $\tau_{m}=0.4$. Moreover, the benefits by fictitiously adjusting the locations of two BSs compared to that for one BS can also be observed in both the LOS and the NLOS environments. With the incorporation of the geometric information into the location estimation, the merits of the proposed GOLE schemes can be observed.

\section{CONCLUSION}

The location estimation algorithms with the assistance from the geometric dilution of precision (GDOP) are presented in this paper. Two GDOP-assisted location estimation (GOLE) algorithms are proposed by considering the geometric layouts

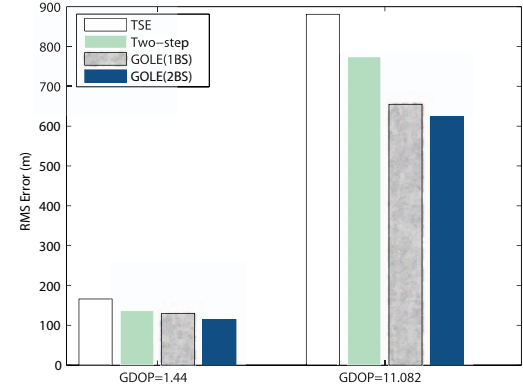

Fig. 4. Performance comparison under the NLOS environment with both better (left: $\mathrm{G}_{\boldsymbol{x}_{k}}^{b}=1.44$ ) and worse (right: $\mathrm{G}_{\boldsymbol{x}_{k}}^{w}=11.08$ ) geometric layouts $\left(\tau_{m}=0.3\right)$.

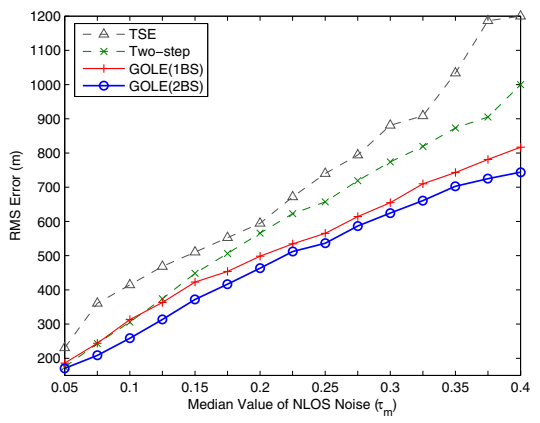

Fig. 5. Performance comparison under the NLOS environment with worse geometric layout $\left(\mathrm{G}_{\boldsymbol{x}_{k}}^{w}=11.08\right)$ : RMS error v.s. median value of NLOS noise.

between the mobile station and its associated base stations (BSs). The GDOP information is utilize to fictitiously relocate the positions of the BSs in order to obtain a better geometric layout for location estimation. It is shown in the simulation results that the proposed GOLE schemes can provide consistent accuracy for location estimation, especially under the environments with poor GDOP values.

\section{REFERENCES}

[1] L. Perusco and K. Michael, "Control, trust, privacy, and security: evaluating location-based services," IEEE Technol. Soc. Mag., vol. 26, no. 1, pp. 4-16, 2007.

[2] N. Patwari, J. Ash, S. Kyperountas, I. Hero, A.O., R. Moses, and N. Correal, "Locating the nodes: cooperative localization in wireless sensor networks," IEEE Signal Processing Mag., vol. 22, no. 4, pp. 54-69, July 2005.

[3] W. Foy, "Position-Location Solutions by Taylor-Series Estimation," IEEE Trans. Aerosp. Electron. Syst., vol. 12, pp. 187-194, March 1976.

[4] K. Chan, Y.T. Ho, "A simple and efficient estimator for hyperbolic location," IEEE Trans. Acoust., Speech, Signal Processing, vol. 42, pp. 1905-1915, Aug 1994.

[5] N. Levanon, "Lowest GDOP in 2-D scenarios," in Proc. IEE Radar, Sonar and Navigatio, vol. 147, June 2000, pp. 149 - 153.

[6] J. Chaffee and J. Abel, "GDOP and the Cramer-Rao bound," in Proc. IEEE Position Location and Navigation Symposium, Las Vegas, NV, Apr. 1994, pp. 663 - 668.

[7] R. J. Kelly, "Reducing geometric dilution of precision using ridge regression," IEEE Trans. Aerosp. Electron. Syst., vol. 26, pp. 154 - 168, Jan. 1990.

[8] P. C. Chen, "A non-line-of-sight error mitigation algorithm in location estimation," in Proc. IEEE Wireless Communications and Networking Conference, vol. 1, Apr. 1999, pp. 316 - 320. 